\title{
Monte Carlo Simulation of Grain Growth During Laser Ablation
}

\author{
Shuang Liu ${ }^{a}, Z_{\text {Znguun Tian }}^{a, *}$, Xuesong GaO ${ }^{b}$ And Deqiao Xie ${ }^{a}$ \\ ${ }^{a}$ Nanjing University of Aeronautics and Astronautics, \\ No. 29, Yudao Str., Qinhuai District, Nanjing City, Jiangsu Province, 210016, China \\ ${ }^{b}$ Shanghai Institute of Optics and Fine Mechanics, Chinese Academy of Sciences, \\ No. 390, Qinghe Road, Jiading District, Shanghai, 201800, China
}

Received: 04.06.2020 \& Accepted: 29.07.2020

Doi: 10.12693/APhysPolA.138.819

*e-mail: tianzj@nuaa.edu.cn

\begin{abstract}
A Monte Carlo model was used to simulate the grain growth during a laser ablation. The effect of temperature distribution on the grain growth was successfully elucidated. In particular, it was observed that the grain size was larger in the middle than at the edges of the system and the evolution process of coarse grains was detected. The grain growth trend depends on temperature distribution.
\end{abstract}

topics: grain growth, Monte Carlo simulation, Gaussian distribution, non-uniform temperature field

\section{Introduction}

It is well known that the grain size is an important parameter that influences material properties. Studies on the simulation of grain growth have become very important $[1,2]$ and have attracted considerable attention in recent years [3-5]. However, very few studies concerned the case of the grain growth in the presence of a non-uniform temperature field with a Gaussian distribution.

The methods widely used to simulate the grain growth have included the cellular automata, the Monte Carlo (MC) and the phase field. The MC method is popular because it requires a small number of iterations and it has higher computational efficiency than the phase field and cellular automata methods. Anderson et al. [6, 7] first used the MC simulation in 1983 to determine the grain growth in a 2D model. Miyake [8] used the Monte Carlo simulation in 1998 to determine the normal grain growth and the real normal grain growth. It was proved then that the driving force for the entire process was essentially the energy reduction at a grain boundary being a result of the grain boundary migration.

Wang et al. [9] developed in 2007 a new modified transition pro-probability model and demonstrated that the boundary energy and the surface energy are indeed the driving forces for the grain growth. Liu et al. [10] used a modified MC model to simulate the reaction kinetics of the grain growth and the microstructural behavior under various desorption-recombination processing conditions. It was shown that the phase transition during the recombination was driven by the interfacial free energy change of the grain boundary and the Gibbs free energy reduction of the recombination reaction. The grain growth was affected by the energy reduction of the grain boundary due to the coalescence of grains. Maazi [11] determined in 2017 that the relationship between the real time and $t_{\mathrm{MCS}}$ was linear. A new equation instead of the transition probability was used in the MC simulation for modeling the influence of temperature.

The MC simulation has been indicated in many studies, although it has rarely been applied to describe the grain growth of nanoparticles, especially with a non-uniform temperature condition [5]. In this paper, the MC model is used to simulate the grain growth during a non-uniform temperature field with the Gaussian distribution. The process of grain growth is observed during a laser ablation.

\section{MC simulation and Gaussian mathematical model}

\subsection{MC simulation of grain growth}

The MC method with salient features has been widely applied for grain growth modelling and has been described in [12-15]. For this reason, we do not repeat these details here. The MC simulation uses a discrete lattice to create a continuum microstructure. The number of each lattice's orientation is random - its value is between 1 and $Q$ (representing the crystal-lattice orientation), where $Q$ is the total number of grain orientations. One can estimate the location of the grain boundary between two adjacent lattice sites based on the crystallographic orientation. Two lattice sites with 
different orientations having a boundary segment are assumed to be different grains, while two adjacent sites with similar orientations are assumed to be the same grain. The grain growth is generally considered to be the result of the grain boundary migration which is driven by a reduction in the system energy. Thus, the nucleation and growth of the grains are simulated by the transformation and reorientation of the lattice elements. The grain boundary energy is determined by defining the interaction between the nearest-neighbor lattice sites. The local interaction energy $(E)$ is given with the Hamiltonian

$$
E=-J \sum_{<i j>}^{n}\left(\delta_{s_{i}, s_{j}}-1\right)
$$

where $J$ is the positive coupling constant that determines the scale of the grain boundary energy, the symbols $s_{i}$ and $s_{j}=1,2, \ldots, Q$ represents the grain orientation in the nearest neighbor lattice sites $i$ and $j$, the angular brackets denote summation over the nearest neighbor lattice pairs and $\delta_{k, l}$ is the Kronecker delta function. The sum is determined over all neighboring sites in the specified "control volume" around the model unit that is inspected. The nearest-neighbor pairs give a nonzero contribution to the system energy if having an opposite orientation. This is ensured by the Kronecker delta function defined as:

$$
\delta_{s_{i}, s_{j}}=\left\{\begin{array}{lll}
1 & \text { if } & s_{i}=s_{j} \\
0 & \text { if } & s_{i} \neq s_{j}
\end{array} .\right.
$$

The probability of reorientation is given by

$$
w=\left\{\begin{array}{ll}
1 & \text { for } \quad \Delta E \leq 0 \\
\mathrm{e}^{-\Delta E /\left(k_{\mathrm{B}} T\right)} & \text { for } \Delta E>0
\end{array},\right.
$$

where $\Delta E$ is the energy change, $T$ is the absolute temperature and $k_{\mathrm{B}}$ is the Boltzmann constant. A successful transition at a grain boundary, i.e., changing in orientation to the nearest-neighboring grain orientation, corresponds to boundary migration [16]. The absorption of energy at a position for which the temperature is equal to $T_{1}$ is described by the expression

$$
E_{\text {laser }}=n T_{1},
$$

where $n$ is the coefficient.

Now, the driving force of the grain growth will be the reduction of both the interfacial free energy at the grain boundary and the energy provided by the laser. Namely,

$$
\begin{aligned}
& \Delta E=J\left[\sum_{\langle i j\rangle}\left(1-\delta_{s_{i}, s_{j}}\right)-\sum_{\langle i j\rangle}\left(1-\delta_{s_{i}, s_{j}}^{\circ}\right)\right] \\
& +\Delta E_{\text {laser. }}
\end{aligned}
$$

The symbol ${ }^{\circ}$ denotes the grain orientation before the change. In turn,

$$
\Delta E_{\text {laser }}=n\left(T_{1}-T_{1}^{\prime}\right),
$$

where $T_{1}$ and $T_{1}^{\prime}$ are temperatures of two adjacent grains separately.
We followed the idea of Wang et al. [9] and introduced a modification to the transformation probability $w$. As a result, we dealt with the expression

$$
\widetilde{w}=\frac{1-\tanh \left(\Delta E /\left(k_{\mathrm{B}} T_{0}\right)\right.}{2\left(1+\exp \left(-\alpha \frac{\left(T-T_{0}\right)}{T}\right)\right)},
$$

where $\alpha$ is the temperature correction parameter, $T_{0}$ is the Tammann temperature of the system, $\Delta E$ means the change in the system energy (before and after) and $T$ is the simulated temperature. Note that when $T$ approaches $T_{0}$, the grain growth probability $\widetilde{w}$ increases obviously. One can also say that during this period the grains grow fleetly. As the total growth requires migration of the grain boundaries, therefore the diffusion rate of the grain boundaries increases significantly near $T_{0}$. According to the knowledge about the actual sintering process, the grain growth can be observed when the sintering temperature approaches $T_{0}$ which seems consistent with the process described by (7).

\subsection{Temperature distributions induced by irradiation of Gaussian beam}

From the heat conduction equation it follows that the transient temperature distribution of the ceramic coating surface depends on the field temperature. For simplicity of calculations, the thermal absorption of the ceramic coating will be treated as the boundary condition effect when the Gaussian laser beam is vertically incident on the coating. A preliminary study of this kind was performed in [17-19].

The intensity distribution is calculated using

$$
P=\frac{2 P_{0}}{\pi \omega_{a}^{2}} \exp \left(-2 \frac{r^{2}}{\omega_{a}^{2}}\right) .
$$

To obtain the temperature distribution $T_{1}$, we based on the heat conduction equation, the Laplace transformation and the Hankel transformation, thus

$$
T_{1}=\frac{T_{0} P_{0}}{P_{1}} \exp \left(-2 \frac{r^{2}}{\omega_{a}^{2}}\right),
$$

where $r$ is the certain distance, $P_{0}$ is the laser power and represents the initial power, $\omega_{a}$ is the radius of the beam, $T_{0}$ denotes the temperature at the center point and $P_{1}$ is determined with the use of Eq. (2.9) in [17].

\subsection{Modeling parameters}

The microstructures are mapped onto a quadratic lattice with $4 / 8$ nearest neighbors and periodic boundary conditions. Each lattice point represents an $\mathrm{MC}$ unit which has an integer value that represents the orientation $Q$ of the lattice point. The MC model is a $1000 \times 1000$ field in this $2 \mathrm{D}$ simulation. The edge length of the quadratic site is $1 \mathrm{~nm}$ in the simulation domain, the area of each site is $1 \mathrm{~nm}^{2}$ and the corresponding actual grain diameter is $40 \mathrm{~nm}$. The assigned orientation number $Q$ is 800. The simulation time is expressed as the number of the Monte Carlo step (MCS) [16]. Since the ceramic grain system is porous, the problem of 
the intrinsic power-law growth index is a complicated matter. The growth index calculation is not considered in this paper [18].

\section{Results and discussion}

Figure 1 shows the temperature field distribution and energy field distribution of the laser beam [20]. The parameters are as follows: $P_{0}=500 \mathrm{~W}$, $\lambda=1064 \mathrm{~nm}$ and $\omega_{a}=1000 \mathrm{~nm}$. It is possible to obtain the radius of the beam $\omega_{a}$ at a certain distance $r$. A series of measurements were conducted to determine the complete beam profile and thus we were able to calculate the beam radius $\omega_{a}$ for several $r$ positions. Using (8) and (9), we plotted the Gaussian energy distribution and temperature distribution of the laser beam in the MATLAB software and the temperature distribution in the Origin software. Figure 1a and b represent the Gaussian distribution at different defocusing distances and the energy field distribution, respectively. Figure 1c represents the temperature field distribution. The laser energy is concentrated at the top-hat peak and the energy is lower at the edges in Fig. 1a. It is observed that the average intensity of the power decreases with the increase in the defocusing distance in Fig. 1b. As seen in Fig. 1c, the temperature distribution is in agreement with the Gaussian energy distribution. The temperature also decreases with the defocusing distance increasing.

It is assumed that the melting point of the ceramic material equals $2700^{\circ} \mathrm{C}$. Figure 2 shows the simulation results of the grain growth for the Gaussian distribution of the laser beam. The detailed description is as follows. The temperature is $1600^{\circ} \mathrm{C}$ at the center and at the edges it is $400^{\circ} \mathrm{C}$
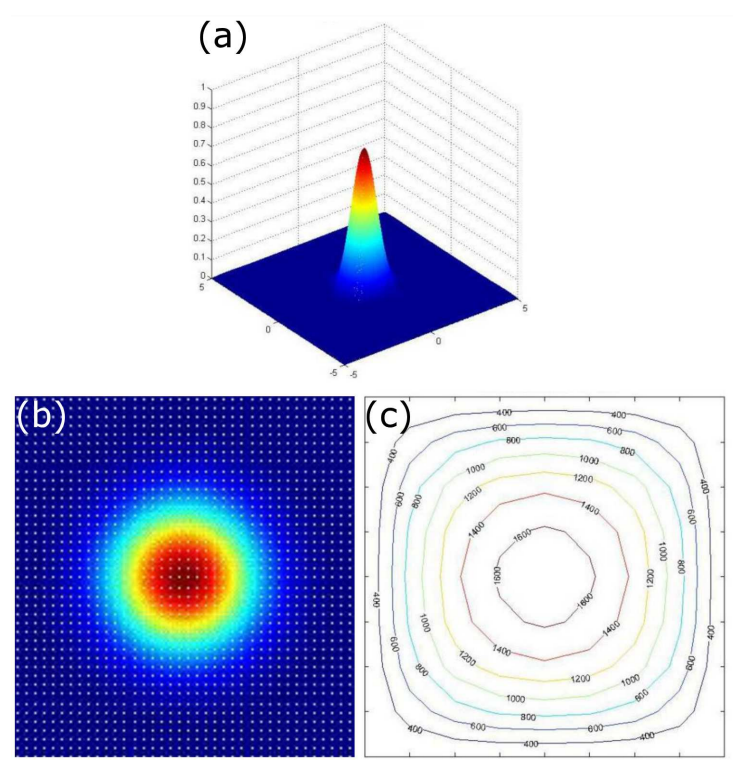

Fig. 1. Temperature field and energy field distribution of laser beam: (a) Gaussian distribution, (b) energy field distribution, (c) temperature field distribution.

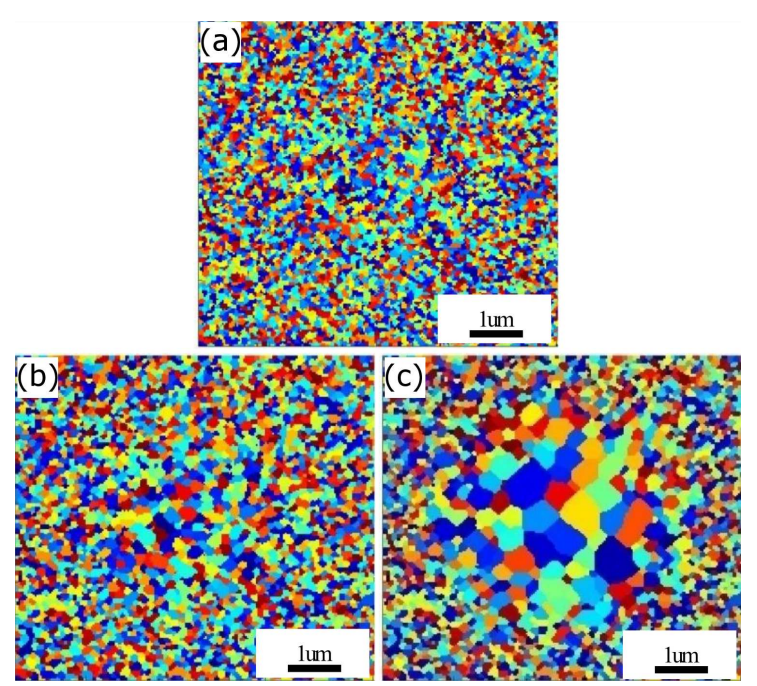

Fig. 2. Grain growth microstructure evolution.

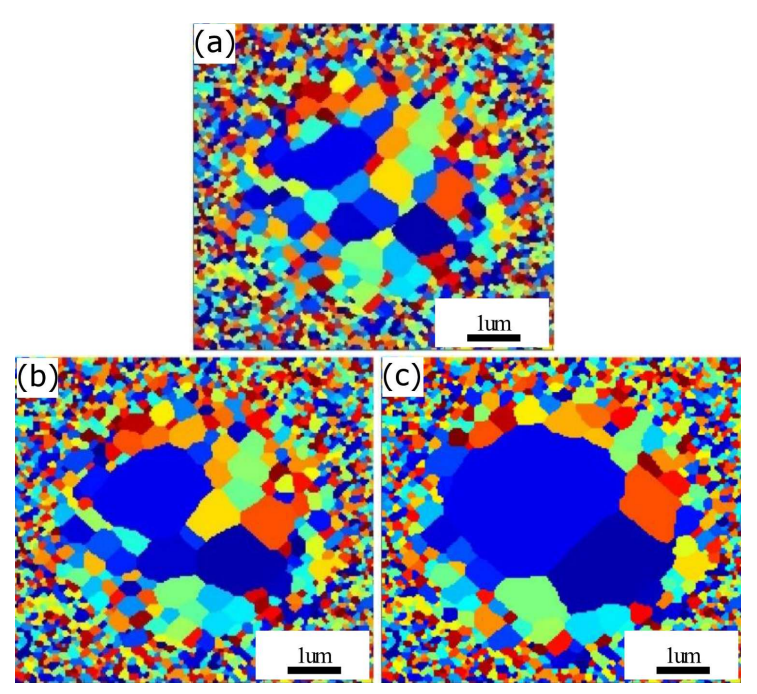

Fig. 3. Grain coarseness microstructure evolution.

below the melting point. In Fig. $2 \mathrm{a}-\mathrm{c}$ one can notice that the grains are extremely large in the center region, while the grains at the edges are very small. A non-isothermal growth occurs in the range of $600-1600^{\circ} \mathrm{C}$. The reason for this is that the energy $\Delta E$ provided by temperature is the driving force of the grain growth and it is absorbed by the grains. The higher is the temperature, the larger amount of energy is absorbed and thus the grain growth occurs more rapidly at higher energy. It is worth to add that the process of the grain growth is affected by large grains assimilating small grains. The kinetic equation of the grain growth is $D^{n}-D_{0}^{n}=k t$, where $k$ is the fitting parameters, $D$ is the average grain size, $t$ is the time and $D_{0}$ is the initial grain size. If $D \gg D_{0}$, the kinetic equation of the grain growth can be expressed as $D^{n}=k t$ [21-24].

The reduction of the interfacial energy is closely related to a shrinkage of the grain boundaries. The laser ablation provides a higher energy, thus interfacial energy increases. This extra energy is used 


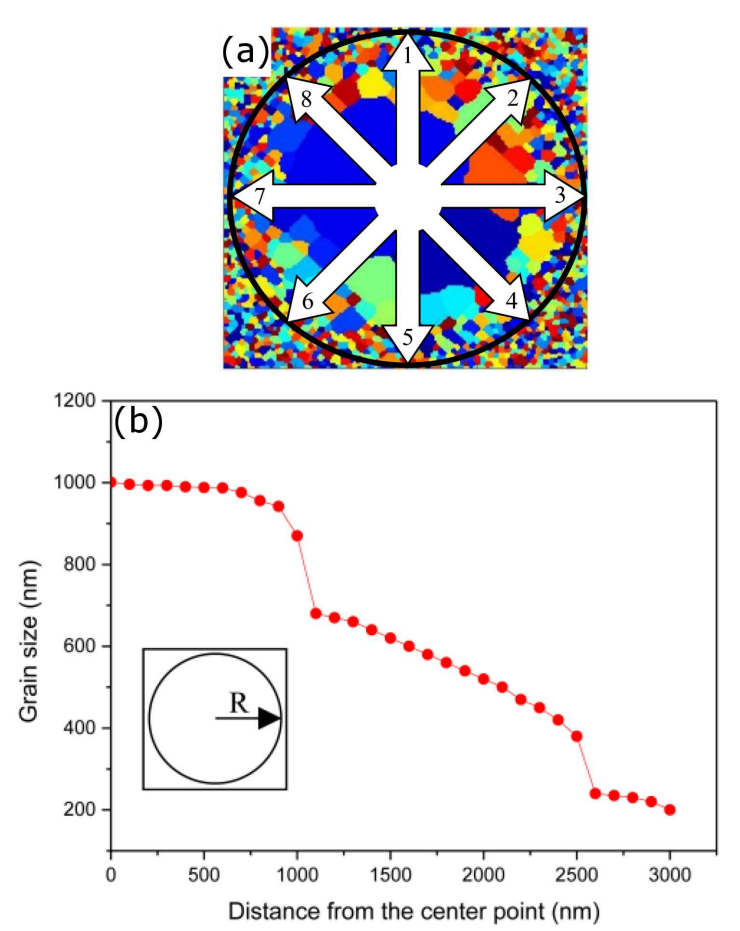

Fig. 4. Grain coarseness microstructure evolution.

TABLE I

The value of the grain size depending on the given direction (distance from the center is $1.5 \mu \mathrm{m}$ ).

\begin{tabular}{c|c|c|c|c}
\hline \hline Direction & 1 & 2 & 3 & 4 \\
\hline Grain size $[\mathrm{nm}]$ & 502.64 & 605.26 & 765.81 & 1011.84 \\
\hline Direction & 5 & 6 & 7 & 8 \\
\hline Grain size $[\mathrm{nm}]$ & 864.62 & 452.32 & 512.52 & 495.31
\end{tabular}

as the driving force of the grain growth for a long time, causing the grains to become coarse. Therefore, as shown in Fig. 3, relatively coarse grains appear. The grain coarsening process results in the grain sizes from $1 \mu \mathrm{m}$ to $3 \mu \mathrm{m}$ (see Fig. $3 \mathrm{a}-\mathrm{c}$ ). From $[25,26]$ we also know that the coarsening of grains creates a boundary fracture and promotes the formation of microcracks at the coating surface $[25,26]$. Then, the coating is subsequently damaged by the laser. This, in fact, could be essential for the basic studies on the laser damage mechanism in long-term laser ablation. The result would provide guidance for the optimal selection of the technological parameters [27-29].

The temporal evolution of the grain size as a function of the distance from the center is presented in Fig. 4. The grain size at different directions is different, while the distance from the center remains constant. In this specific example, the value of the grain size distance from the center is $2 \mu \mathrm{m}$. It can be seen in Table I that the direction is different and the grain size is different. The grain size of direction 1 is $500 \mathrm{~nm}$ and of direction 4 $1000 \mathrm{~nm}$. Therefore, it is necessary to take the average value of eight directions. The average value

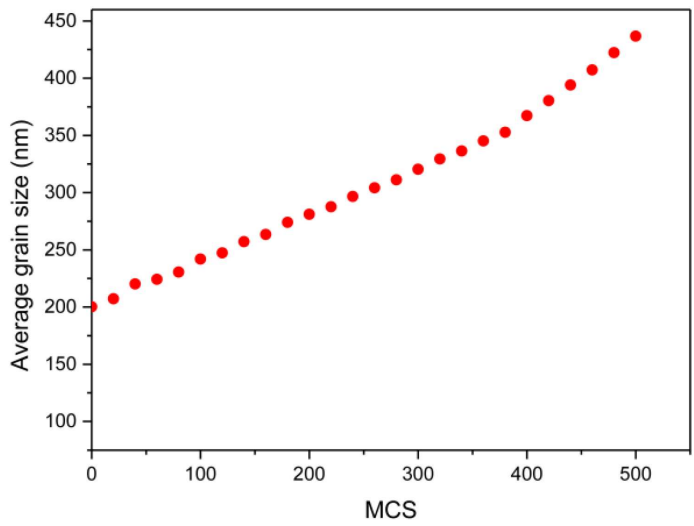

Fig. 5. The average grain size vs. MCS steps.

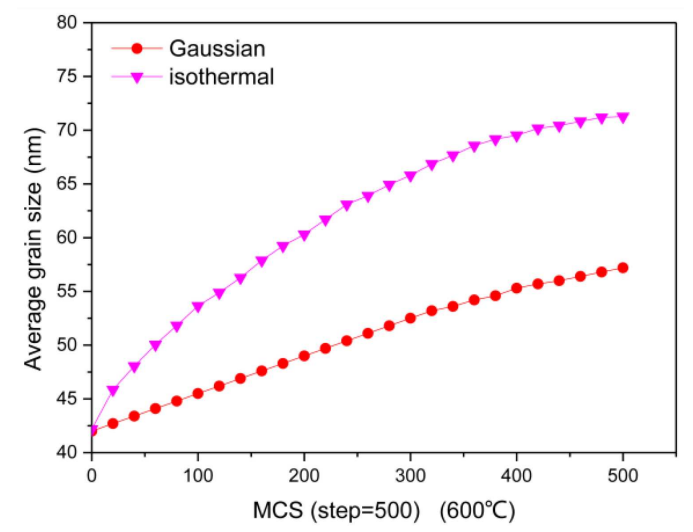

Fig. 6. The grain growth tendency at Gaussian distribution and isothermal distribution.

of the eight directions is calculated in Fig. 4a and shown in Fig. 4b. Note that the decreasing trend is steady when $R$ changes from 0 to $1 \mu \mathrm{m}$. The reason for that is the appearance of the coarse grain whose size reaches about $1 \mu \mathrm{m}$. The grain size presents fluctuation and the decreasing trend is obvious from 1 to $2.5 \mu \mathrm{m}$. It is caused by the fact that the energy changes with the distance from the center. There is no fluctuation in the grain size in the range from 2.5 to $3 \mu \mathrm{m}$. The reasons for that are a low interfacial energy and no extra energy. The grain growth process is illustrated in Fig. 5. The average grain size tends to increase with increasing MCS; it grows rapidly at 400-500 MCS.

Figure 6 shows the grain growth for the Gaussian beam distribution and isothermal distribution at the conditions of $600^{\circ} \mathrm{C}$ and 500 MCS. The grain growth was found more rapid in isothermal condition than in the Gaussian distribution. The reason for this is that in the center region it is the Gaussian distribution that assures $T=600^{\circ} \mathrm{C}$, while in the entire region beyond - it is the isothermal condition. The grain growth is normal under isothermal condition and because of that the final grain size is the largest [30]. The underlying explanation for this observation is the temperature distribution which impacts the grain growth trend. 


\section{Conclusion}

Three conclusions can be drawn based on our study:

1. The modified MC method is used to simulate the microstructural evolution and the mechanism of grain growth of nanostructured ceramic materials during a laser ablation.

2. It turns out that the grain growth is rapid at high temperatures. The temperature distribution impacts the grain growth trend. The rule of influence of temperature on normal grain growth is broken.

3. The driving force of the grain growth is the reduction of the interfacial energy and it is related to the shrinkage of the grain boundaries, whereas the laser ablation provides extra energy which leads to the occurrence of the coarse grain. The coarse grains cause microcrack generation, hence the coating is damaged. The coarse grain is one of the laser damage mechanisms.

\section{Acknowledgments}

Project (51605473) was supported by the Young Scientists Fund of the National Natural Science Foundation of China; Project (BK20161476) was supported by the Jiangsu Provincial Research Foundation for Basic Research, China and Project (BE2015029) was supported by the Science and Technology Planning Project of Jiangsu Province of China.

\section{References}

[1] W.W. Liu, C.Y. Wei, K. Yi, J.D. Hao, Chin. Opt. Lett. 13, 62 (2015).

[2] J. Liu, Y.L. Qiao, P. Zhang, Y.C. Xue, Z. Cai, Surf. Coat. Technol. 321, 491 (2017).

[3] X.L. Ling, X.F. Liu, G. Wang, Z.X. Fan, Vacuum 119, 145 (2015).

[4] S. Mishra, T. DebRoy, Mater. Sci. Technol. 22, 253 (2013).

[5] F. Baino, M.A. Montealegre, G. Orlygsson, G. Novajra, C. Vitale-Brovarone, J. Mater. Sci. 52, 1 (2017).

[6] M.P. Anderson, D.J. Srolovitz, G.S. Grest, P.S. Sahni, Acta Metall. 32, 783 (1984).

[7] D.J. Srolovitz, G.S. Grest, M.P. Anderson, Acta Metall. 34, 1833 (1986).
[8] A. Miyake, Contrib. Mineral. Petrol. 130, 121 (1998).

[9] H.D. Wang, H. Zhang, H.L. Li, Y.C. Tang, Chin. J. Nonferr. Metal. 17, 990 (2007).

[10] X.Y. Liu, X. Wang, H.F. Sun, L.X. Hu, Trans. Nonferr. Metal. Soc. Chin. 21, 412 (2011).

[11] N. Maazi, Adv. Math. Phys. 31, 1 (2017).

[12] K. Ito, ISIJ Int. 57, 1625 (2017).

[13] P.E. Goins, E.A. Holm, Comput. Mater. Sci. 124, 411 (2016).

[14] I.N. Karkin, L.E. Karkina, P.A. Korzhavyi, Y.N. Gornostyrev, Phys. Solid State 59, 106 (2017).

[15] D. Zöllner, P. Streitenberger, Mater. Sci. Forum 567, 81 (2008).

[16] X. Liu, L. Hu, Comput. Mater. Sci. 67, 417 (2013).

[17] K. Takamoto, S. Nakayama, J. Jpn. Soc. Prec. Eng. 443, 901 (1971).

[18] M.R. Dudek, J.F. Gouyet, M. Kolb, Surf. Sci. 401, 220 (1998).

[19] A. Sharma, M.S. Sodha, S. Misra, S.K. Mishra, Laser Part. Beams 31, 403 (2013).

[20] Z.L. Horváth, Z. Bor, Opt. Commun. 222 , 51 (2003).

[21] H.Y. Ma, C.L. Mo, S.P. Du, Adv. Mater. 538, 869 (2012).

[22] S. Sista, T. Debroy, Metall. Mater. Trans. 32, 1195 (2001).

[23] H.D. Wang, Z. Hai, H.L. Li, Y.C. Tang, Chin. J. Nonferr. Metal. 17, 990 (2007).

[24] Y. Liu, L.F. Cheng, Q.F. Zeng, Z.Q. Feng, J. Zhang, J.H. Peng, C.W. Xie, K. Guan, Mater. Des. 55, 740 (2014).

[25] P. Blikstein, A.P. Tschiptschin, Cryst. Res. Technol. 29, 99 (1999).

[26] H. Ravash, L. Vanherpe, J. Vleugels, N. Moelans, J. Europ. Ceram. Soc. 37, 2265 (2017).

[27] F. Wakai, Mater. Sci. Forum 539, 2359 (2007).

[28] Y. Zhao, H. Zhang, H. Wei, Q. Zheng, T. Jin, Acta Metall. Sin. 49, 981 (2013).

[29] Y. Suwa, ISIJ Int. 52, 582 (2012).

[30] D. Tian, Q.Q. Shen, X. Gao, L.C. Li, Y.L. Zhao, B.S. Xu, H.S. Jia, Mater. Mech. Eng. 35, 100 (2011). 